\title{
Novos registros de ferrugens (fungi, Uredinales) para o Brasil, coletados no Parque Nacional do Itatiaia
}

\author{
Mauricio Salazar Yepes ${ }^{1,2}$ e Aníbal Alves de Carvalho Júnior ${ }^{3,4}$
}

Recebido 28/05/2009. Aceito 1/02/2010

RESUMO - (Novos registros de ferrugens (fungi, Uredinales) para o Brasil, coletados no Parque Nacional do Itatiaia). A partir de recentes coletas de Uredinales realizadas no Parque Nacional do Itatiaia e em áreas de proteção ambiental ao redor deste parque, foram identificadas novas ocorrências para o Brasil: Dicheirinia binata (Berkeley \& Curtis) Arthur, Maravalia manettiae Jørstad, Prospodium bignoniacearum (Spegazzini) Cummins, Puccinia ancizari Mayor, Puccinia investita Schweinitz, Puccinia lasiacidis Kern, Puccinia mandevillae Jackson \& Holway e Uredo chusqueae Pardo-Cardona. Estas espécies já haviam sido reportadas em alguns países adjacentes ao Brasil. Além disso, após minuciosa análise bibliográfica, é proposta a sinonimização de Puccinia interjecta Jackson para Puccinia ancizari Mayor. Estas coletas permitiram incrementar as coleções brasileiras e as coleções do Herbário do Instituto de Pesquisas Jardim Botânico do Rio de Janeiro (RB).

Palavras-chave: ferrugem, hospedeiro, PARNA Itatiaia, Mata Atlântica

ABSTRACT - (New rust fungi records (Uredinales) from Brasil, collected at the Itatiaia National Park). From recent collections of Uredinales undertaken at Itatiaia National Park and in environmental protection areas around this park, new records for Brazil were identified: Dicheirinia binata (Berkeley \& Curtis) Arthur, Maravalia manettiae Jørstad, Prospodium bignoniacearum ( Spegazzini) Cummins, Puccinia ancizari Mayor, Puccinia investita Schweinitz, Puccinia lasiacidis Kern, Puccinia mandevillae Jackson \& Holway and Uredo chusqueae Pardo-Cardona. These species had been reported in several countries adjacent to Brazil. Moreover, after careful literature analysis, synonymization of Puccinia interjecta Jackson to Puccinia ancizari Mayor is proposed. These collections are increasing the Brazilian specimens and those of the of the Instituto de Pesquisas Jardim Botânico do Rio de Janeiro Herbarium (RB). Key words: rust fungi, host, PARNA Itatiaia, Atlantic forest

\section{Introdução}

A leste do território brasileiro destaca-se como principal paisagem o bioma da Mata Atlântica. O regime de chuvas, a temperatura e a colonização histórica pelas plantas promoveram o aparecimento de um perfil altitudinal e microclimático, influenciando decisivamente na sua biodiversidade. Nesta região destaca-se o Parque Nacional do Itatiaia por suas características únicas e, particularmente, pela grande diversidade vegetal e seu alto grau de endemismo (Safford 1999).

Neste contexto, o conhecimento dos fitopatógenos é de grande importância no estabelecimento de programas de manejo, tanto de áreas degradadas como naturais. Entre os fitopatógenos de plantas nativas e exóticas estão os fungos da Ordem Uredinales, também denominados ferrugens. Estes fungos são parasitos obrigados, altamente especializados aos hospedeiros, muito diversos e mundialmente dispersos, tendo cerca de 120 gêneros e 6000 espécies (Cummins \& Hiratsuka 2003). No Brasil, a Ordem Uredinales está representada por 56 holomorfos, nove anamorfos e cerca de 800 espécies (Hennen et al. 2005). A partir de levantamento bibliográfico e de alguns herbários constatou-se que havia registros de 36 espécies de Uredinales, entre elas oito tipos para o Parque Nacional do Itatiaia. Estas espécies estavam parasitando 37 espécies de plantas pertencentes a 15 famílias botânicas.

Coletas mais recentes estão permitindo realizar um incremento nas coleções brasileiras de ferrugens e, além disso, possibilitando melhorar a interpretação sobre a biologia, ecologia e biogeografia deste importante grupo de parasitos de plantas. O objetivo deste trabalho é apresentar as novas ocorrências de Uredinales para o Brasil, coletadas durante os levantamentos realizados por período de dois anos, pelo projeto "Uredobiota (fungi) do Parque Nacional do Itatiaia".

\section{Material e métodos}

Foram realizadas coletas bimensais, com duração de cinco dias, por período de 2 anos, entre 2006 e 2008, no Parque Nacional do Itatiaia, e em áreas de proteção ambiental ao redor do parque, nos estados de Minas Gerais e Rio de Janeiro, entre os paralelos $22^{\circ} 19^{\prime}$ e $22^{\circ} 45^{\prime}$ latitude sul e os medianos $44^{\circ} 15^{\prime}$ ' e $44^{\circ} 50^{\prime}$ ' de longitude W (Parque Nacional do Itatiaia, 2006), numa faixa de altitude entre $587-2600 \mathrm{~m}$. Para isso foi observada praticamente toda vegetação adjacente às estradas e trilhas, com curtas incursões no interior da mata. Foram examinadas, em detalhe, todas as partes superficiais das plantas, procurando-se os sintomas e sinais do patógeno, identificando-se preliminarmente os hospedeiros e as ferrugens, quando possível. Os exemplares suspeitos foram coletados, herborizados e levados ao Laboratório de Micologia do Instituto de Pesquisas Jardim Botânico do Rio de Janeiro para a identificação definitiva dos hospedeiros e das ferrugens. Foram observados sintomas e sinais, realizadas raspagens e cortes, assim como montadas lâminas em lacto fenol e hidrato de cloral sob estereomicroscópio Leica S6E. Para a observação das estruturas microscópicas das ferrugens foi utilizado microscópio Zeiss "Axioskop 40", acoplado à câmera digital Zeiss "Axiocam MRc". Foi utilizado o programa Axiovision Rel. 4.6. para captura de imagens e medição dos esporos. As ferrugens foram identificadas pelos caracteres morfológicos, gama de hospedeiros e comparação com a literatura especializada na Ordem Uredinales.

\section{Resultados e Discussão}

1. Dicheirinia binata (Berkeley \& Curtis) Arthur, N. Am. Flora 7: 147. 1907. (0/Ipe,IIpe/III). Tipo sobre Fabaceae indeterminada: NICARAGUA. s.loc., s.d. e s.col.

\footnotetext{
Universidad Nacional de Colombia, Medellín, Colombia

2 Instituto de Pesquisas Jardim Botânico do Rio de Janeiro, Programa de Pós Graduação em Botânica, Rio de Janeiro, RJ, Brasil

3 Instituto de Pesquisas do Jardim Botânico do Rio de Janeiro, Rio de Janeiro, RJ, Brasil

4 Autor para correspondência: anibal@jbrj.gov.br
} 
$=$ Triphragmium binatum Berkeley \& Curtis, Proc. Am. Acad. 4: 125. 1858.

$=$ Diorchidium binatum De Toni, in Saccardo, Syll. Fung. 7: 736. 1888.

Anamorfo: Lecythea pezizaeformis Berkeley \& Curtis, Proc. Am. Acad. 4: 127. 1858.

$=$ Uredo pezizaeformis De Toni, in Saccardo, Syll. Fung. 7: 856. 1888.

$=$ Uredo cabreriana Kern \& Kellerman, J. Myc. 13:25. 1907.

Fig. 1-2

Espermogônios associados a galhas, epífilos, marrons-canela escuros. Écios em Uredo associados aos espermogônios. Uredínios em Uredo, soros anfígenos, caulícolos, confluentes, ruptura da epiderme conspícua, irrompentes, pulverulentos, de origem subepidermal, marrons-canela; paráfises ausentes; esporos obovóides, globosos, globoso-clavados, 24-30 x 24-27 $\mu \mathrm{m}$; parede equinulada, 2-3 $\mu \mathrm{m}$ de espessura, sendo um pouco menor no ponto de inserção do pedicelo, de cor castanhodourada; poros germinativos 3-4 equatoriais, raramente supraequatoriais.

Material examinado: Erythrina sp.(Fabaceae): BRASIL. Rio de Janeiro: Itatiaia, 20/X/2006, [II], M. Salazar Yepes et al. 369-06 (RB).

Distribuição geográfica: América Central, América do Sul e Caribe (Cummins 1978) e Brasil.

Ciclo de vida: Autóico.
Télio não encontrado no material coletado no PARNA Itatiaia. Segundo Arthur (1907), tem soros hipófilos, parecidos com o anamorfo, porém escuros; esporos bicelulares, amplamente obovóides, 35-40 x 26-29 $\mu \mathrm{m}$, planos ou arredondados no ápice e reduzidos na base; parede coberta por projeções, mais ou menos ramificadas, até $12 \mu \mathrm{m}$ de comprimento, $2-3 \mu \mathrm{m}$ de espessura lateral, marrom-canela; pedicelo fino, persistente, hialino, mais ou menos do comprimento do esporo.

Espécies cultivadas do gênero Erythrina, comumente usadas como ornamentais, podem apresentar problemas sérios causados pelo ataque de $D$. binata, diminuindo seus atributos ornamentais.

2. Maravalia manettiae Jørstad, Ark. Bot. (Ser.2) 4: 85 . 1959. (0/Icv,IIpe/III). Tipo sobre Manettia sp.: EQUADOR. Entre Alaspungo e Gualea, 22/V/1920, I. A. Holmgren s.n.

Fig. 3-7

Espermogônios raros, epífilos, em áreas cloróticas, marrom escuros. Écios em Aecidium, soros epífilos, agrupados ao redor dos espermogônios; células do perídio raras, retangulares, verrugosas; esporos globosos, obovóides, oblongos, 27-36 x 24-30 $\mu \mathrm{m}$; parede com verrugas de até 3 $\mu \mathrm{m}$ de comprimento misturadas com áreas lisas, 1-1,5 $\mu \mathrm{m}$ de espessura, hialina; poros germinativos não visíveis. Uredínios em Uredo, soros hipófilos, pequenos, arredondados,
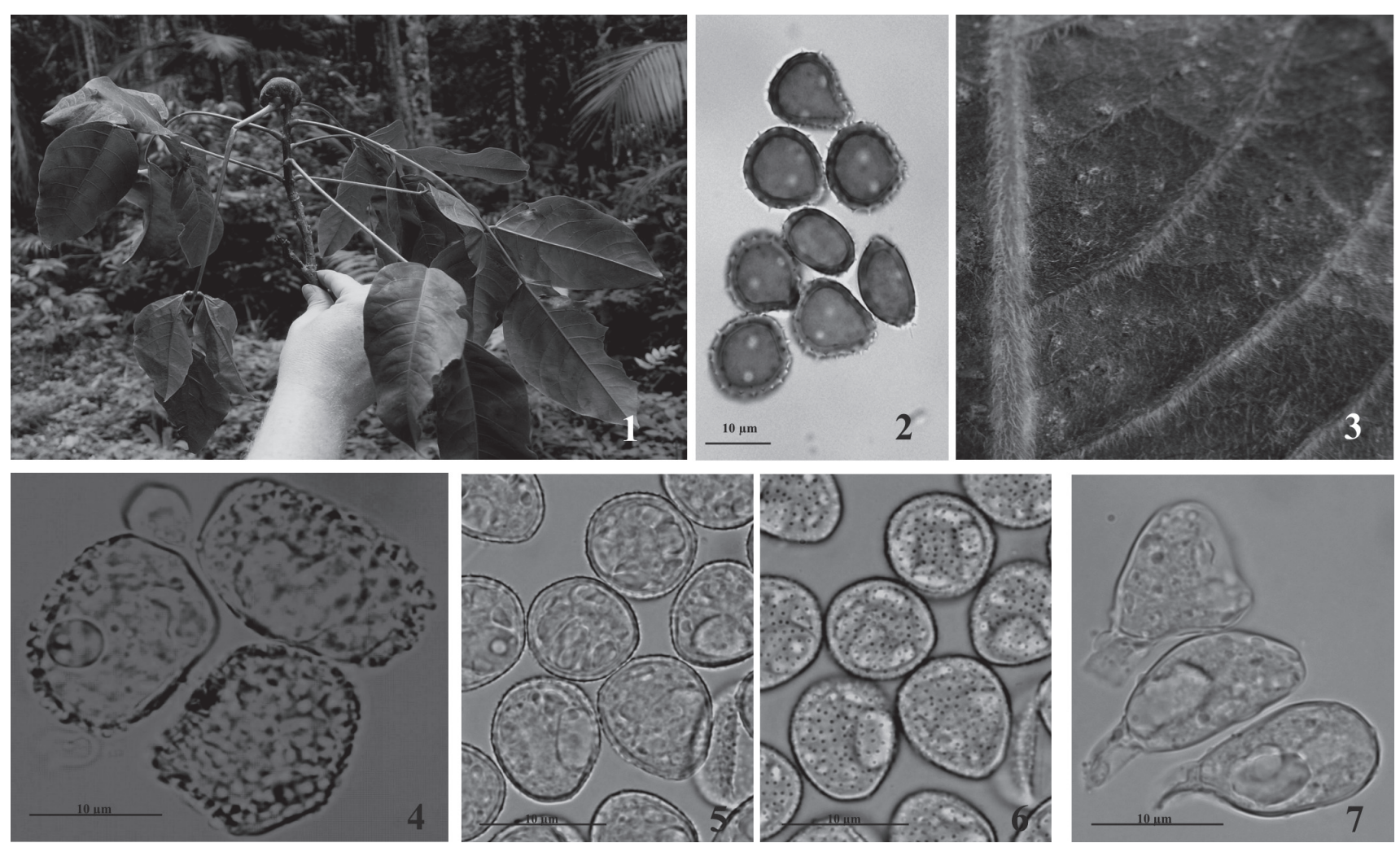

Figuras 1-2. Dicheirinia binata (Berkeley \& Curtis) Arthur: 1. Sintomas da ferrugem. 2. Esporos do uredínio em vista mediana. Figuras 3-7. Maravalia manettiae Jørstad: 3. Sintomas da ferrugem. 4. Esporos do écio em vista mediana. 5. Esporos do uredínio em vista mediana. 6. Esporos do uredínio em vista superficial. 7. Teliósporos. 
solitários ou agrupados, em áreas cloróticas, pulverulentos, de origem subepidermal; paráfises ausentes; esporos obovóides, elipsóides; 27-33 x 18-24 $\mu$ m; parede com equínulas finas e uniformemente dispostas, de hialina a amarela pálida; poros germinativos não visíveis. Télios com soros hipófilos, solitários, pequenos, compactos, de origem subepidermal, de brancos a amarelos pálidos; esporos elipsóides, clavados, 33-45 x 15-18 $\mu \mathrm{m}$; parede lisa, 0,5-1 $\mu \mathrm{m}$ de espessura, uniforme, hialina; poro germinativo não visível; pedicelo hialino, uma vez o comprimento do esporo.

Material examinado: Manettia congesta (Vell.) Schw. (Rubiaceae): BRASIL. Minas Gerais: Itamonte, 25/ IV/2007, [II/III], M. Salazar Yepes et al. 299-07 (RB). Rio de Janeiro: Itatiaia, 16/II/2007, [II/III], M. Salazar Yepes et al. 220-07 (RB); 11/VI/2007, [II/III], M. Salazar Yepes \& A.A. Carvalho Jr. 383-07 (RB). Manettia sp.: BRASIL. Minas Gerais: Itamonte, 04/XII/2006, [II/III], M. Salazar Yepes et al. 416-06 (RB); 16/VIII/2006, [II], M. Salazar Yepes et al. 157-06 (RB); 17/X/2006, [II/III], M. Salazar Yepes et al. 302-06 (RB); 25/IV/2007, [II/III], M. Salazar Yepes et al. 305-07 (RB); 12/VI/2007, [II/III], M. Salazar Yepes \& A.A. Carvalho Jr. 402-07 (RB); 12/VI/2007, [II], M. Salazar Yepes \& A.A. Carvalho Jr. 404-07 (RB). Rio de Janeiro: Itatiaia, 07/VIII/2007, [0/I/II/III], M. Salazar Yepes \& A.A. Carvalho Jr. 511-07 (RB); 10/VIII/2007, [0/I/ II/III], M. Salazar Yepes \& A.A. Carvalho Jr. 556-07 (RB); 10/VIII/2007, [II/III], M. Salazar Yepes \& A.A. Carvalho Jr. 561-07 (RB); 09/X/2007, [II/III], M. Salazar Yepes \& A.A. Carvalho Jr. 605-07 (RB).

Distribuição geográfica: Equador (Jørstad, 1959) e Brasil.

Ciclo de vida: Autóico.

No Brasil existe o registro de Uromyces tener Schroeter sobre Manettia gracilis Chamisso \& Schlectendahl, coletada em Santa Catarina. Apesar de ser microcíclica, o teleomorfo dessa espécie é muito semelhante ao teleomorfo de $M$. manettiae (Jørstad 1959). Portanto, a revisão do material Tipo ajudaria a esclarecer os questionamentos feitos por Jørstad (1959).

\section{Prospodium bignoniacearum (Spegazzini) Cummins,} Lloydia 3: 58. 1940. (?/?,IIpe/III). Tipo sobre Clytostoma callistegioides (Cham.) Bureau ex Griseb. (na publicação como Bignonia sp.): PARAGUAI. Guarapi: X/1883, Balansa-3932.

$\equiv$ Puccinia bignoniacearum Spegazzini, Anal. Soc. Cient. Argentina 26: 11. 1888.

Anamorfo: Uredo bignoniacearum Spegazzini, Anal. Soc. Cient. Argentina 26: 14. 1888.

Fig. 8-12

Uredínios com soros hipófilos, solitários ou agrupados, pequenos, arredondados, saindo pelos estômatos, pulverulentos, marrons-canela; paráfises cilíndricas, encurvadas, agudas no ápice, flexuosas, ao redor do soro, 24-36 x 6-9 $\mu \mathrm{m}$; parede de cor castanho-dourada pálida a amarela pálida, raramente engrossada no ápice, 5-8 $\mu \mathrm{m}$ de espessura; esporos globosos, 24-30 $\mu \mathrm{m}$ de diâmetro, esporos obovóides ou triangulares, 24-30 x 21-24 $\mu \mathrm{m}$; parede bilaminar, lâmina interna com $2 \mu \mathrm{m}$ de espessura, marrom-canela e externa com 1-3 $\mu \mathrm{m}$ de espessura, hialina; 2 poros germinativos equatoriais. Télios com soros hipófilos, saindo do uredínio, marrons-canela escuros; esporos elipsóides ou oblongoelipsóides, 30-36 x 21-24 $\mu \mathrm{m}$; parede com verrugas finas e uniformemente dispostas, bilaminada, $2 \mu \mathrm{m}$ de espessura lateral e 2-3 $\mu \mathrm{m}$ de espessura apical, marrom-canela; poro germinativo da célula basal próximo à inserção do pedicelo e da célula distal apical; pedicelo amarelo pálido, mais ou menos do comprimento do esporo, frequentemente de inserção lateral, com presença ou não de ornamentação.

Material examinado: Bignoniaceae indeterminada: BRASIL. Rio de Janeiro: Itatiaia, 12/X/2007, [II/III], $M$. Salazar Yepes \& A.A. Carvalho Jr. 640-07 (RB).

Distribuição geográfica: Paraguai e Uruguai (Cummins 1940) e Brasil.

Ciclo de vida: Desconhecido.

Este hospedeiro é uma liana da família das Bignoniaceae que, devido a ausência de caracteres reprodutivos não foi possível identificar ao nível de gênero.

4. Puccinia ancizari Mayor, Mém. Soc. Neuchatel Sci. Nat. 5: 525. 1913. (0/Icv,IIpe/III). Tipo sobre Baccharis nitida Pers.: COLOMBIA. Cundinamarca: caminho entre Villeta e Facatativá, 2000 m, [I/III], 09/X/1910, Mayor 065.

= Puccinia interjecta Jackson \& Holway, Mycologia 24: 148. 1932. nom. nudum.

= Puccinia ancizari Arthur, N. Amer. Fl. 7: 476. 1921. nom. nudum.

$=$ Allodus ancizari (Mayor) Arthur \& Orton., N. Amer. Fl. 7: 476. 1921.

Anamorfo: Caeoma ancizari Salazar \& Buriticá, Rev. Fac. Nac. Agron. Medellín 61(1): 4291. 2008.

Aeciure ancizari Salazar \& Buriticá Rev. Fac. Nac. Agron. Medellín 61(1): 4292. 2008.

Fig. 13-18

Espermogônios epífilos, raros, agrupados em pequenas áreas necróticas, marrons-canela. Écios em Caeoma, soros hipófilos, opostos aos espermogônios, poucos, cobertos pela epiderme e abertos por um poro, de origem subepidermal, arredondados, pulverulentos, amarelo pálidos; esporos catenulados, elipsóides, obovóides, globosos, angulosos, 33-42 x 21-33 $\mu \mathrm{m}$; parede verrugosa, $1-3 \mu \mathrm{m}$ de espessura uniforme, de amarela pálida a hialina; poros germinativos não visíveis. Uredínios em Aeciure, soros anfígenos, solitários ou agrupados, de origem subepidermal, ruptura da epiderme por um poro, pulverulentos, marrons-canela; paráfises ausentes; esporos obovóides, amplamente elipsóides, 30-39 x 24-27 $\mu$ m; parede com equinulas finas e uniformemente dispostas, 1-2 $\mu \mathrm{m}$ de espessura uniforme, hialina; poros germinativos não 

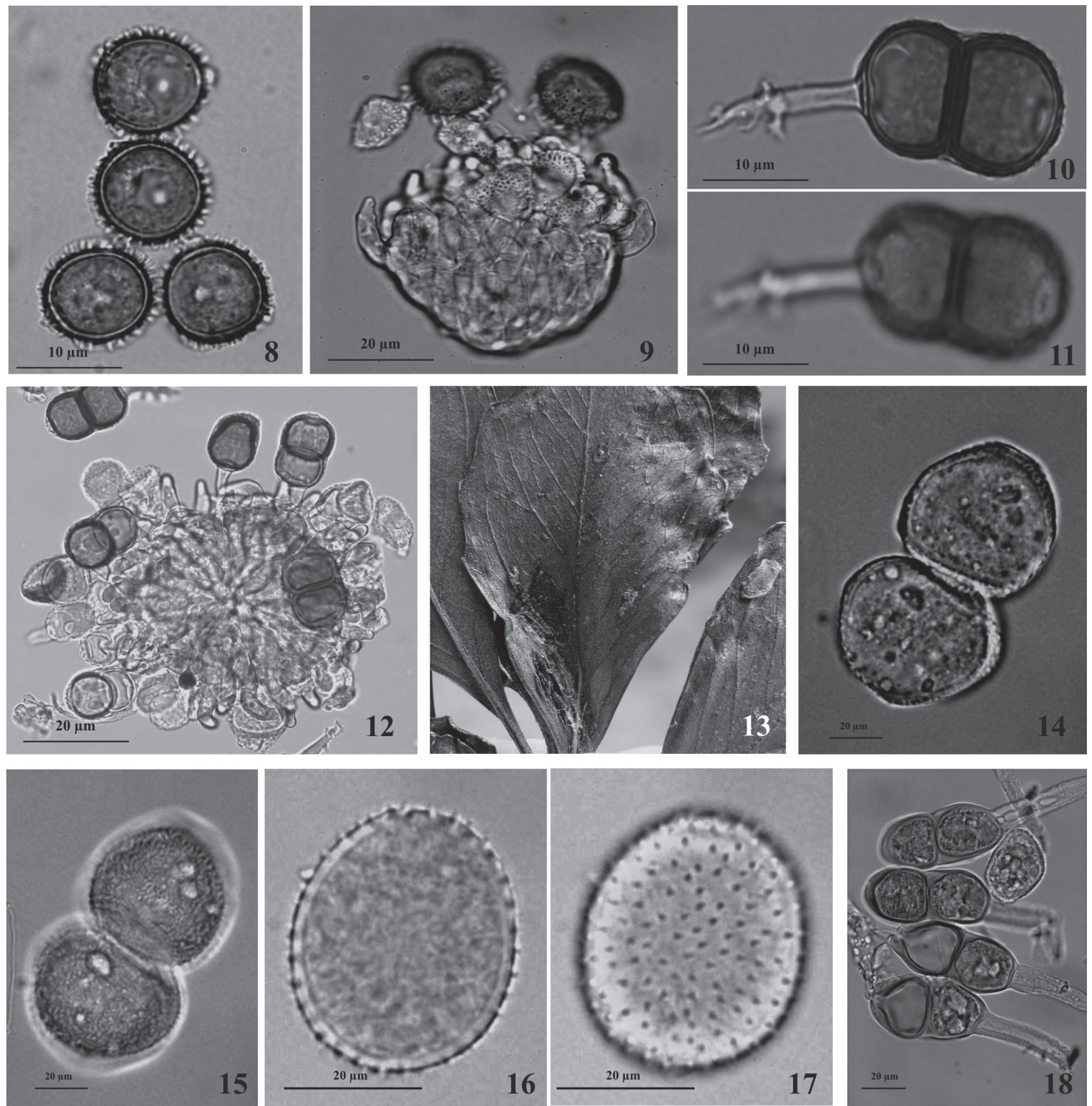

Figuras 8-12. Prospodium bignoniacearum (Spegazzini) Cummins: 8. Esporos do uredínio em vista mediana. 9. Aspecto geral do soro uredinial mostrando as paráfises características da espécie. 10. Teliósporos em vista mediana. 11. Teliósporos em vista superficial. 12. Aspecto geral do soro telial mostrando as paráfises características da espécie. Figuras 13-18. Puccinia ancizari Mayor: 13. Sintomas da ferrugem. 14. Esporos do écio em vista mediana. 15. Esporos do écio em vista superficial. 16. Esporos do uredínio em vista mediana. 17. Esporos do uredínio em vista superficial. 18. Teliósporos.

visíveis. Télios com soros anfígenos, predominantemente hipófilos, solitários ou raramente agrupados, arredondados, pequenos, ruptura da epiderme conspícua, irrompentes, pulvinados, marrons-canela e acinzentados quando germinados; esporos elipsóides, clavados, 48-72 x 21-33 $\mu \mathrm{m}$, constritos no septo; parede lisa, 1-2 $\mu \mathrm{m}$ de espessura lateral e 3-6 $\mu \mathrm{m}$ de espessura apical, de amarelo-dourada a castanho-dourada; poro germinativo da célula basal próximo do septo e célula da distal, apical; pedicelo hialino, com até duas vezes o comprimento do esporo, parede engrossada, até $3 \mu \mathrm{m}$ de espessura.

Material examinado: Baccharis platypoda DC.(Asteraceae): BRASIL. Minas Gerais: 15/II/2007, [0/I/III], M. Salazar Yepes \& A.A. Carvalho Jr. 100-07 (RB). Rio de Janeiro: Itatiaia, 07/VIII/2007, [II/III], M. Salazar Yepes \& A.A. Carvalho Jr. 513-07 (RB); 09/X/2007, [0/I/II/III], M. Salazar Yepes \& A.A. Carvalho Jr. 609-07 (RB). 
Distribuição geográfica: Colômbia e Guatemala (Arthur, 1918) e Brasil.

Ciclo de vida: Autóico.

Existe certa confusão na literatura: Mayor (1913) publicou a nova espécie $P$. ancizari Mayor sobre Baccharis nitida Pers. de material proveniente da Colômbia (Mayor 65), descrevendo o écio como verrugoso. Arthur (1918), estudando materiais provenientes da Guatemala, identificou Puccinia ancizari Mayor (Holway 103, 660). Arthur (1921) sugeriu a nova combinação Allodus ancizari (Mayor) Arthur \& Orton a partir do mesmo material coletado na Guatemala, entretanto, descreveu o écio como equinulado. Posteriormente, o gênero Allodus Arthur foi considerado sinônimo de Puccinia uma vez que era baseado em diferenças apenas no tipo de ciclo de vida. Jackson (1932), entendendo que o material estudado por Arthur (1921), na verdade era diferente do material de Mayor (1913) por apresentarem diferenças na ornamentação do écio, sugeriu erroneamente o nome Puccinia interjecta Jackson como uma nova combinação para Puccinia ancizari Arthur (não $P$. ancizari Mayor). Entretanto, a espécie P. ancizari Arthur não foi publicada formalmente (nom. nudum). Posteriormente, Salazar \& Buriticá (2008), estudando amostras coletadas na Colômbia sobre Baccharis nitida Pers., publicaram Caeoma ancizari (écios-verrugoso) e Aeciure ancizari (uredínio-equinulado) como anamorfos de $P$. ancizari Mayor, complementando em parte a descrição de Mayor. A partir das coletas efetuadas no Parque Nacional do Itatiaia todos os estádios esporíferos da espécie foram encontrados e são descritos conjuntamente pela primeira vez. São dados os sinônimos dos teleomorfos e dos anamorfos e conclui-se que, na verdade, $P$. interjecta Jackson é sinônimo de $P$. ancizari Mayor.

5. Puccinia investita Schweinitz Trans. Am. Phil. Soc. Ser. 2 4(2): 296. 1832. (0/Icv,IIce/III). Neotipo sobre Gnaphalium sp.: ESTADOS UNIDOS DA AMÉRICA. Nova York: Long Island, 20/X/1905, Farlow (PUR 36470; isotipo Reliq. Farl. No. 258).

= Dicaeoma investitum (Schweinitz) Kuntze, Revis. gen. pl. (Leipzig) 3: 469. 1898.

= Puccinia gnaphaliata (Schweinitz) Arthur \& Bisby, Proc. Am. Phil. Soc. 57: 221. 1918.

Anamorfo: Aecidium trixiphillum Speg., Bol. Acad. Nac. Cs. De Córdoba. 29: 150. 1926.

$=$ Caeoma gnaphaliatum Schweinitz, Trans. Am. Phil. Soc. II, 4; 292.1832.

Fig. 19-27

Écio em Aecidium, soros hipófilos, caulícolos, dispersos, cupulados, 1-3 mm de comprimento, brancos; células do perídio fortemente unidas, de retangulares a poliédricas, 45$60 \times 24-27 \mu \mathrm{m}$, parede externa verrugosa e interna estriada, hialina; esporos globosos, elipsóides ou angulares; parede finamente verrugosa, 1-3 $\mu \mathrm{m}$ de espessura, de hialina a amarela pálida. Uredínios em Aeciure, soros epífilos, solitários ou em pequenos grupos, poucos, pequenos, arredondados, cobertos pela epiderme, depois abertos por um poro central, pulverulentos, de origem subepidermal; paráfises ausentes; esporos oblongo-elipsóides, globosos ou obovóides, 27-33 x 24-27 $\mu \mathrm{m}$; parede equinulada, $1-2 \mu \mathrm{m}$ de espessura uniforme, amarela pálida; poros germinativos 6-8 dispersos. Télios com soros anfígenos, solitários, raros, arredondados, irrompentes, pulvinados, descobertos, de cor castanha escura; paráfises ausentes; esporos clavados ou elipsóides, 39-51 x 21-27 $\mu \mathrm{m}$, não constritos no septo; parede lisa, $0,5-1,5 \mu \mathrm{m}$ de espessura lateral e 6-9 $\mu \mathrm{m}$ de espessura apical, marrom-canela; pedicelo decíduo, amarelo pálido.

Material examinado: Pseudognaphalium cheranthifolium (Lam.) Hilliard \& B.L. Burtt.(Asteraceae): BRASIL. Rio de Janeiro: Itatiaia, 14/II/2007, [I], M. Salazar Yepes \& A.A. Carvalho Jr. 76-07 (RB); 20/II/2008, [I/II/III], M. Salazar Yepes \& A.A. Carvalho Jr. 78-08 (RB). Achyrocline sp.(Asteraceae): BRASIL. Minas Gerais: 24/IV/2007, [I/ III], M. Salazar Yepes et al. 279-07 (RB).

Distribuição geográfica: Américas (Cummins, 1978) e Brasil.

Ciclo de vida: Autóico.

Espermogônios não encontrados no material coletado no PARNA Itatiaia. Segundo Cummins (1978), espermogônios epífilos. O écio catenulado e equinulado está sendo descrito pela primeira vez. Com isto estão sendo complementadas as descrições de todos os estádios esporíferos.

6. Puccinia lasiacidis Kern, Mycologia 30: 546. 1938. (?/?,IIpe/III). Tipo sobre Lasiacis divaricata (L.) Hitchc.: VENEZUELA. Caracas: Chaco, reserva na fazenda dos Dominguez, 18/IV/1934, Kern \& Toro 1718. Fig. 28-31

Uredínio em Uredo, soros hipófilos, em áreas necróticas, solitários ou agrupados, pequenos, ao longo das nervuras, de origem subepidermal, ruptura da epiderme conspícua, pulverulentos, marrom-canela; paráfises cilíndricas ou capitadas, 39-51 x 3-12 $\mu \mathrm{m}$; parede amarela pálida ou castanho-dourada pálida, esporos obovóides, 24-30 x 18-24 $\mu \mathrm{m}$; parede equinulada, 1-1,5 $\mu \mathrm{m}$ de espessura uniforme, de cor castanho-dourada; poros germinativos 3-5 equatoriais. Télios com soros hipófilos, solitários, pequenos, de origem subepidermal, irrompentes, pulvinados, de coloração marrom-canela escura; esporos oblongos ou oblongo-elipsóides, 21-30 x 18-21 $\mu \mathrm{m}$; parede lisa, 1-2 $\mu \mathrm{m}$ de espessura lateral e 2-4 $\mu \mathrm{m}$ de espessura apical, de cor castanho-dourada; poros germinativos não visíveis; pedicelo amarelo pálido, freqüentemente de inserção lateral, mais ou menos duas ou três vezes o comprimento do esporo.

Material examinado: Lasiacis sp.(Poaceae): BRASIL. Rio de Janeiro: Itatiaia, 14/VII/2006, [II], M. Salazar Yepes et al. 88-06 (RB); 17/VIII/2006, [II], M. Salazar Yepes et al. 223-06 (RB); 18/VIII/2006, [II], M. Salazar Yepes et al. 239-06, 241-06 (RB); 05/XII/2007, [II/III], M. Salazar Yepes \& A.A. Carvalho Jr. 717-07 (RB). 

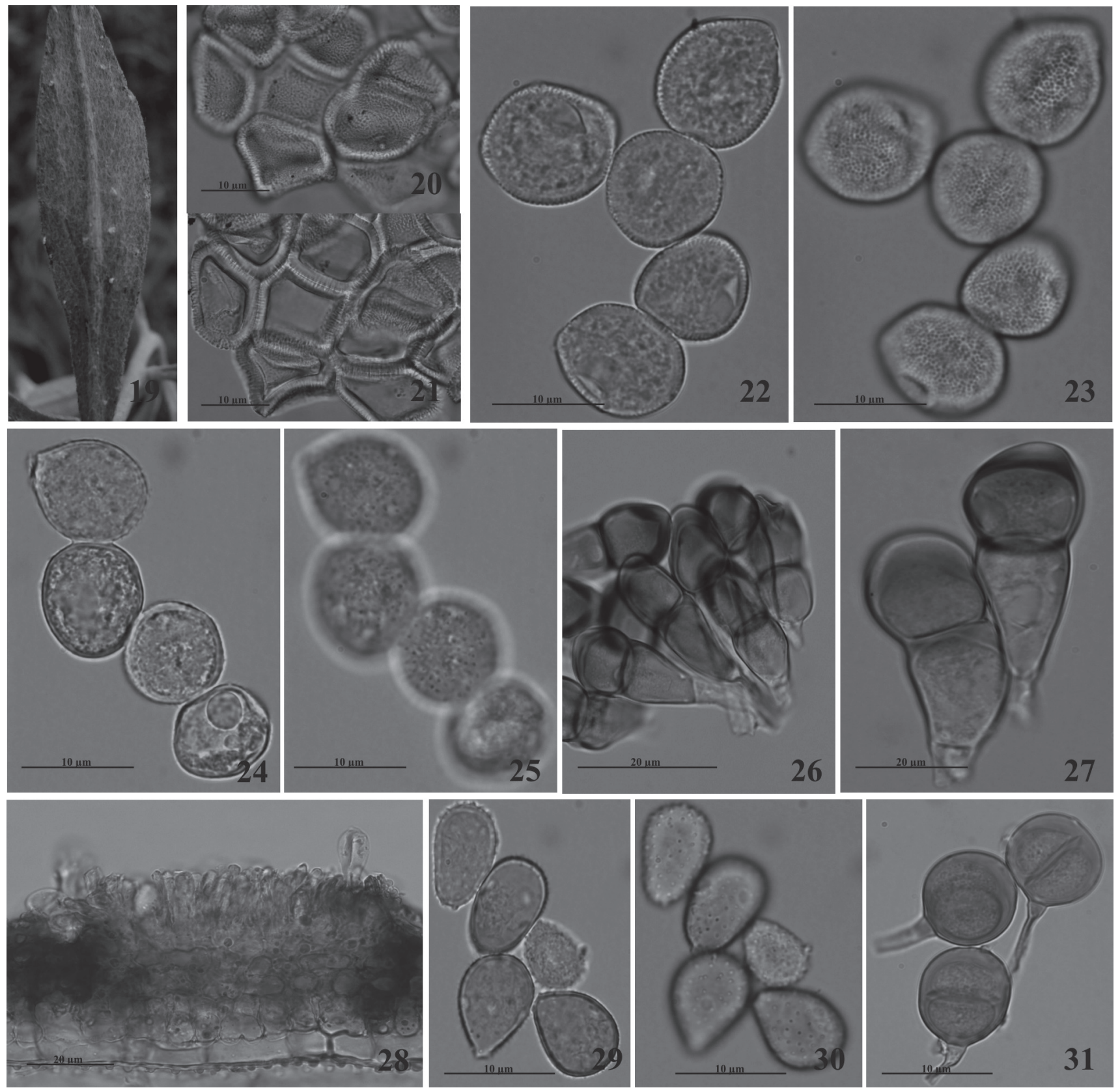

Figuras 19-27. Puccinia investita Schweinitz: 19. Sintomas da ferrugem. 20. Células do perídio em vista mediana. 21. Células do perídio em vista superficial. 22. Esporos do écio em vista mediana. 23. Esporos écio em vista superficial. 24. Esporos do uredínio em vista mediana. 25. Esporos do uredínio em vista superficial. 26. Teliósporos. 27. Detalhe dos Teliósporos em vista mediana mostrando o engrossamento das paredes. Figuras 28-31. Puccinia lasiacidis Kern: 28. Aspecto geral do soro com a presença de muitas paráfises. 29. Esporos do uredínio em vista mediana. 30. Esporos do uredínio em vista superficial. 31. Teliósporos.

Distribuição geográfica: Venezuela (Cummins 1971) e Brasil.

Ciclo de vida: Desconhecido.

A espécie P. lasiacidis é diferente de Uromyces costaricensis e Phakopsora lenticularis, também coletadas sobre Lasiacis, por apresentar esporos bicelulares típicos do gênero Puccinia. Apesar de na descrição original não constarem paráfises, algumas amostras por nós estudadas as apresentaram, esporadicamente, nos soros do uredínio. Entretanto, outras características como dimensões, ornamentações e poros germinativos dos esporos nos levaram a identificar as amostras como P. lasiacidis.

7. Puccinia mandevillae Jackson \& Holway, in Jackson, Mycologia 23: 492. 1931. (?/?,?/III). Tipo sobre Mandevilla mandoni Rusby: BOLÍVIA. Sorata: 19/IV/1920, Holway-556.

Fig. 32-34

Télios com soros anfigenos, predominantemente hipófilos, em áreas cloróticas, 0,4-1 mm de diâmetro, agrupados em cír- 
culos concêntricos, pulvinados, compactos, ruptura da epiderme inconspícua, marrom-canela escura, quando germinados acinzentados; paráfises ausentes; esporos elipsóides, obovóides, amplamente elipsóides, 24-33 x 18-21 $\mu \mathrm{m}$, septo oblíquo comumente encontrado; parede lisa, 2-3 $\mu \mathrm{m}$ de espessura uniforme, marromcanela; poro germinativo não visível; pedicelo persistente, 39-105 $\mu \mathrm{m}$ de comprimento, de cor canela pálida, freqüentemente de inserção lateral. Mesosporos presentes, raros.

Material examinado: Mandevilla sp.(Apocynaceae): BRASIL. Rio de Janeiro: Itatiaia, 12/IV/2007, [III], $M$. Salazar Yepes \& A.A. Carvalho Jr. 05-07 (RB); 23/IV/2007, [III], M. Salazar Yepes et al. 190-07 (RB); 23/IV/2007, [III], M. Salazar Yepes et al. 233-07 (RB); 14/IV/2008, [III], M. Salazar Yepes \& A.A. Carvalho Jr. 132-08 (RB).

Distribuição geográfica: Bolívia e Colômbia (Buriticá \& Pardo-Cardona, 1996) e Brasil.

Ciclo de vida: Microcíclico.
Esta espécie é muito semelhante a $P$. cynanchi reportada sobre a família Asclepiadaceae (Hennen et al. 2005). Como atualmente Mandevilla e outros gêneros de Asclepiadaceae foram incluídos em Apocynaceae, talvez seja conveniente o desenvolvimento de trabalho de revisão das espécies $P$. cynanchi e $P$. mandevillae, com o objetivo de determinar se realmente trata-se de espécies distintas. No caso de sinonimização, $P$. cynanchi tem prioridade por ter sido descrita anteriormente por Berkeley \& Curtis em 1853.

8. Uredo chusqueae Pardo-Cardona, Rev. Acad. Colombiana Cienc. 20(77): 205. 1996. (?/?,IIpe/?). Tipo sobre Chusquea serrulata Pilger: COLÔMBIA. Antioquia, Abejorral, ao redor da ponte sobre o rio Quebradona, estrada via Sonsón, 2100 m, 15/X/1992, Pardo-Cardona 238, MMUNM 475.

Fig. 35-38
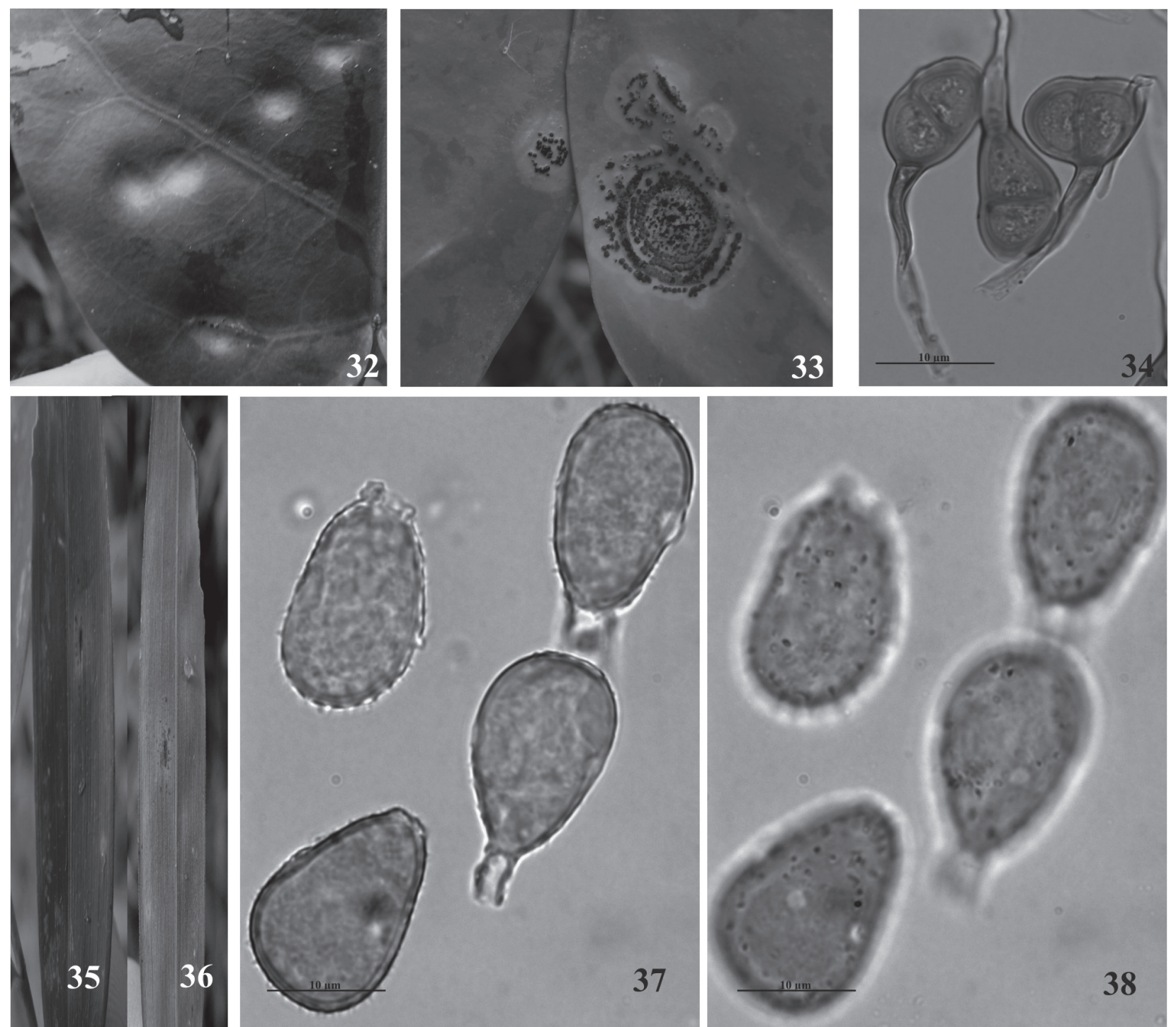

Figuras 32-34. Puccinia mandevillae Jackson \& Holway: 32. Sintomas da ferrugem no lado adaxial. 33. Sintomas e sinais da ferrugem no lado abaxial. 34. Teliósporos. Figuras 35-38. Uredo chusqueae Pardo-Cardona: 35. Sintomas da ferrugem no lado adaxial. 36. Sintomas e sinais da ferrugem no lado abaxial. 37. Esporos do uredínio em vista mediana. 38. Esporos do uredínio em vista superficial. 
Uredínio em Uredo, soros hipófilos, solitários ou agrupados, pequenos, ao longo das nervuras, em áreas necróticas, ruptura da epiderme conspícua, pulverulentos, de coloração marrom-canela; paráfises raras ou ausentes, capitadas, pequenas, até $27 \mu \mathrm{m}$, de hialinas a castanhas pálidas; esporos obovóides ou oblongo-elipsóides, 24-33 x 18-24 $\mu \mathrm{m}$; parede com equínulas finas e uniformemente dispostas, 1-2 $\mu \mathrm{m}$ de espessura uniforme, de amarela pálida a castanho-dourada; poros germinativos 2-5 equatoriais.

Material examinado: Chusquea sp: BRASIL. Minas Gerais: 06/XII/2006, [II], M. Salazar Yepes \& A.A. CarvaIho Jr. 471-06 (RB); Itamonte, 17/X/2006, [II], M. Salazar Yepes et al. 298-06 (RB); 20/II/2008, [II], M. Salazar Yepes \& A.A. Carvalho Jr. 58-08 (RB). Rio de Janeiro: Itatiaia, 14/VIII/2006, [II], M. Salazar Yepes et al. 114-06 (RB); 23/ IV/2007, [II], M. Salazar Yepes et al. 214-07 (RB).

Distribuição geográfica: Colômbia (Buriticá \& PardoCardona 1996) e Brasil.

Ciclo de vida: Desconhecido.

$\mathrm{Na}$ descrição de Pardo-Cardona (Buriticá \& PardoCardona 1996) não são descritas paráfises. Entretanto, no material examinado do PARNA Itatiaia esporadicamente elas foram encontradas. Por outro lado, outras características da amostra são muito semelhantes às descritas por aquele autor. Portanto não encontramos razões suficientes para separá-las.

\section{Agradecimentos}

Este trabalho faz parte do projeto de Doutorado realizado pelo primeiro autor no Programa de Pós-Graduação em Botânica, Instituto de Pesquisas Jardim Botânico do Rio de Janeiro. Os autores agradecem ao Parque Nacional do Itatiaia (IBAMA) pelo apoio durante a realização do trabalho de campo; ao Instituto de Pesquisas Jardim Botânico do Rio de Janeiro pela infra-estrutura e apoio logístico; aos botânicos, Carine Garcia Pinto Quinet, Mário Gomes, Fabrício Moreira Ferreira e Rafael Augusto Xavier
Borges, pelo auxilio na identificação das espécies hospedeiras. Ao $\mathrm{CNPq}$ e a FAPERJ pelo apoio financeiro.

\section{Referências bibliográficas}

Arthur, J. C. 1907. Aecidiaceae. North American Flora 7(Part 2): 129-160.

Arthur, J. C. 1918. Uredinales of Guatemala Based on Collections by E. W. D. Holway. IV. Puccinia on Carduaceae, Form-Genera, and Índex. American Journal of Botany 5(10): 522-550.

Arthur, J.C. 1921. Aecidiaceae (continuatio). North American Flora 7(Part 6): 405-480.

Buriticá, P. \& Pardo-Cardona, V. M. 1996. Flora Uredineana Colombiana. Revista de la Academia Colombiana de Ciencias Exactas, Fisicas y Naturales 20(77): 183-236

Cummins, G. B. 1940. The genus Prospodium (Uredinales). Lloydia 3: $1-78$.

Cummins, G. B. 1971. The rust Fungi of Cereals, Grasses and Bamboos. New York Springer-Verlag.

Cummins, G. B. 1978. Rust fungi on legumes and composites in North America. Tucson, Univ. Ariz. Press.

Cummins, G. B., \& Hiratsuka, Y. 2003. Illustrated genera of rust fungi. Thrid Edition. American Phytophatological Society. St Paul.

Hennen, J. F.; Figueiredo, M. B.; Carvalho Júnior, A. A. \& Hennen, P. G. 2005. Catalogue of species of plant rust fungi (Uredinales) of Brazil. Disponível: http://www.jbrj.gov.br/em publicações/publicações gerais. Consultado em 01/03/2007.

Jackson, H. S. 1932. The Rusts of South America Based on the Holway Collections VI. Mycologia 24: 62-186.

Jørstad, I. 1959. Uredinales from South America and tropical North America, chiefly collected by Swedish botanists II. Arkiv for Botanik ser. 2, 4: 49-103.

Mayor, E. 1913. Contribution a l'etude de Uredineés de Colombie. Mémoires de la Societé des Sciences Nattureles de Neuchatel 5 442-599.

Parque Nacional do Itatiaia. 2006. Disponível: http://www.ibama.gov.br/ parna itatiaia. Consultado em 08/09/2008.

Safford, H. D. 1999. Brazilian páramos I. An introduction to the physical environment and vegetation of the campos de altitude. Journal of Biogeography. 26: 693-712.

Salazar, Y. M. \& Buriticá, P. 2008. Nuevas especies para la Uredobiota neotropical. Revista de la Facultad Nacional de Agronomia, Medellín 61(1): 4291-4301. 